\title{
Mejoramiento del tiempo y el espacio en la gestión de un centro quirúrgico: el caso de Botucatu, Brasil
}

\section{Time and space improvement in the management of a surgical center: the case of Botucatu, Brazil}

\author{
Luciana Gisele Bordinhão Duma \\ FATEC-Osasco \\ luciana.duma@fatec.sp.gov.br \\ Fernando de Almeida Santos ${ }^{\mathrm{ii*}}$ \\ Pontificia Universidade Católica de São Paulo \\ almeidasantos@pucsp.br \\ Gabriela Ajani Venturoso \\ FATEC-Osasco \\ gabriela.venturoso@fatec.sp.gov.br \\ José Carlos Trindade Filhoiii \\ Universidade Estadual Paulista Júlio de Mesquita Filho \\ jcarlos@fmb.unesp.br
}

* Autor corresponsal.

Faculdade de Tecnologia de Osasco - Prefeito Hirant Sanazar, R. Pedro Rissato, 30 - Vila dos Remedios, Osasco, SP, BRASIL, CEP: 06296-220.

ii Pontifícia Universidade Católica de São Paulo, Rua Ministro de Codoy, 969, $4^{\circ}$ andar, Sala 4E-06 CEP, São Paulo, BRASIL, CEP: 05015901.

iii Hospital das Clinicas da Faculdade de Medicina de Botucatu- UNESP, Av. Prof. Mário Rubens Guimarães Montenegro s/n., Botucatu, SP, BRASIL, CEP: 18618-687.

\section{Resumen}

El presente estudio tiene como objetivo desarrollar una estructura de indicadores para el monitoreo, la evaluación general y el control del funcionamiento del Centro Quirúrgico del Hospital Clínico de la Escuela de Medicina de Botucatu (HCFMB), el cual busca optimizar el tiempo y mejorar el uso del espacio. El artículo consta de un estudio de caso cualitativo, con la utilización de un cuestionario distribuido y aplicado al equipo del Centro Quirúrgico HCFMB. Se recopilan datos a través de un grupo focal para confirmar qué indicadores se ajustan al Centro Quirúrgico Botucatu HC. Luego, se rechazan algunos indicadores que no se ajustan a la realidad del hospital y se determina que los indicadores relacionados con el tiempo son más relevantes, mientras que los indicadores de desempeño no son importantes. El aporte principal consiste en la elaboración de una propuesta, puesto que, la investigación sobre la temática es escasa, siendo un área muy importante y costosa para los hospitales.

\section{INFORMACIÓN ARTÍCULO}

Recibido: 29 de Septiembre 2020

Aceptado: 16 de Diciembre 2020
Palabras Claves:

Indicadores

Centro Quirúrgico

Contraloría

Costos

Gestión hospitalaria 


\begin{abstract}
This study aims to the creation of an indicator framework for monitoring, general evaluation and control of the operation of the Surgical Center of the Clinical Hospital of the Botucatu Medical School (HCFMB), to optimize time and better use the space available. The article consists of a qualitative case study, using a questionnaire, distributed to the team of the HCFMB Surgical Center. Data was collected through a focus group to confirm which indicators fit the Botucatu HC Surgical Center. After the meeting, some indicators that did not fit the reality of the hospital were eliminated. Time usage indicators were more relevant than performance related ones. The main contribution consists in the elaboration of a proposal, as there is very little research on the subject, being a very important and costly area for hospitals.
\end{abstract}

ARTICLE INFO

Received: 29 September 2020

Accepted: 16 December 2020

Keywords:

Indicators

Surgical Center

Controllership

Costs

Hospital management

\section{INTRODUCCIÓN}

En los últimos años, la esperanza de vida en Brasil ha aumentado considerablemente, según el censo del Instituto Brasileiro de Ceografía e Estatística (IBCE). Según Paradella (2018), a partir de los datos del censo, es posible enunciar que en 2050 la población de adultos mayores será mayor al $22.7 \%$ de la población, es decir, que el país se está moviendo hacia un perfil demográfico de envejecimiento. En consecuencia, un mayor número de personas mayores significa una mayor carga de enfermedades crónicas. En 2007, el 72\% de las muertes en Brasil se atribuyó a enfermedades crónicas, cáncer, enfermedad renal, diabetes, entre otras.

La inactividad física es un factor de riesgo para muchas enfermedades como la obesidad y los problemas cardiovasculares. El consumo de alimentos no nutritivos, industrializados y calóricos, sumado a la ausencia de actividad física, son algunas de las causas de obesidad que ya afectan a más del 50\% de la población brasileña, según una investigación hecha por el Sistema de Vigilancia de Factores de Riesgo - Encuesta de Protección de Enfermedades Crónicas por Teléfono (Vigitel, 2017). Asimismo, es sabido que la obesidad está relacionada con muchos tipos de cáncer, problemas ortopédicos, venas varicosas de miembros inferiores, desarrollo de diabetes adulta (tipo 2) y presión arterial alta. Según el Portal del Gobierno de Brasil (2017), fumar es una enfermedad epidémica responsable de aproximadamente 200 000 muertes al año en Brasil, y más de cincuenta enfermedades relacionadas con el tabaco. Fumar está vinculado con muertes por enfermedad coronaria, cáncer, enfermedades cardiovasculares y neurológicas. Según la Organización Mundial de la Salud (OMS, 2017), alrededor de 2.5 millones de personas mueren cada año en todo el mundo debido al consumo excesivo de alcohol (Centro de Informações de Saúde e Alcool, 2018).

Un informe de la OMS (2017), establece que la mayoría de las discapacidades derivadas de estas enfermedades será tratada con procedimientos quirúrgicos, es decir, el aumento de los procedimientos quirúrgicos está relacionado con el aumento de la esperanza de vida y también con la modernización de la medicina quirúrgica. Las estimaciones muestran que 234 millones de cirugías se realizarán cada año en todo el mundo a medida que aumente la longevidad.

Frente a estos datos, la cirugía se convierte en una preocupación mundial porque afecta las políticas públicas y también sirve como advertencia para reorganizar los sistemas de salud.

Este tema, de suma importancia, ha logrado que la Comisión de Constitución y Justicia de la Asamblea Legislativa del Estado de São Paulo (2016) aprobara el proyecto de ley 625/2016, que otorga transparencia a la lista de espera para cirugías en hospitales públicos o bajo el Sistema Único de Saúde (SUS), en el estado de São Paulo (Damasio, 2016). Este proyecto tiene como objetivo hacer que listas de espera para cirugías sean publicadas en Internet y actualizadas cada mes con nombres de pacientes y números de identificación, fecha de espera y posición ocupada.

Este estudio tuvo como objetivo desarrollar una estructura de indicadores para el monitoreo y control de la operación del Centro Quirúrgico de Hospital Clínico de la Escuela de Medicina de Botucatu (HCFMB, 2017), para elaborar una secuencia de los procesos en breve tiempo, óptima utilización 
del espacio y la forma como se distribuyen las cirugías programadas y entender si los materiales disponibles son suficientes para la demanda de cirugías y están organizados de manera eficiente y efectiva.

Según Mastrantonio y Graziano (2002), "la unidad de quirófano es una fuente importante de ingresos para los hospitales" (p. 333), por lo tanto, el desarrollo de programas de garantía de calidad corresponde a una "una necesidad en términos de eficiencia y una obligación bajo un punto de vista ético y moral" (p. 333).

El HCFMB, por su parte, brinda atención a una población de aproximadamente 2 millones de personas, siendo la institución pública más grande vinculada al Sistema Único de Salud en la región. El Complejo Hospitalario comprende el Hospital de Clínicas, los Primeros Auxilios para Niños, los Primeros Auxilios para Adultos, el Servicio de Atención de Alcohol y Drogas (SARAD) y el Hospital Estatal de Botucatu.

EI HCFMB realiza desde atención ambulatoria sencilla, hasta procedimientos y cirugías altamente complejas, atendiendo un total de 570000 consultas ambulatorias/año, 143000 exámenes de imágenes/año, 16000 trasplantes autólogos de riñón, hígado, córnea y médula ósea al año. El servicio de búsqueda de órganos y tejidos y el Banco de Ojos son responsables de la recaudación de fondos en cincuenta ciudades de la región.

Por lo tanto, el objetivo de este trabajo fue construir indicadores para permitir la evaluación general del Centro Quirúrgico del HCFMB y proponer soluciones.

En tanto, la investigación tiene los siguientes objetivos específicos:

- Analizar la organización y operación del Centro Quirúrgico y las áreas circundantes, indicando dentro del proceso operativo qué indicadores necesitan ser monitoreados para aumentar la efectividad y eficiencia del sector, contribuyendo al aumento en el número de cirugías.

- Seleccionar y validar indicadores para optimizar la sala de operaciones y el número de citas.

Los indicadores están diseñados para satisfacer la necesidad de la sala de operaciones para que cumpla con sus objetivos y metas, mientras que, la selección de estos indicadores es relevante para observar el desempeño a lo largo del tiempo. Se debe evaluar la configuración interna de la sala de operaciones en cuanto a lo que se debe medir para lograr los objetivos propuestos.

Se observa que el estudio de los indicadores de salud es una tendencia creciente que apunta a optimizar los procesos de trabajo, especialmente en quirófanos, y demuestra ser una preocupación mundial.

\section{REFERENCIAL TEÓRICO}

La gestión efectiva significa llevar a cabo una gestión organizacional para que las tareas se completen independientemente de cualquier factor, como el tiempo, el costo y la mano de obra. A su vez, el concepto organizacional permite minimizar los costos.

Desde la mirada de Mastrantonio y Graziano (2002), la forma de lograr el equilibrio entre eficiencia y efectividad en un centro quirúrgico requiere que los gerentes obtengan un conocimiento detallado de la realidad, desde los puntos relacionados con el desarrollo del acto anestésico quirúrgico, hasta el proceso de trabajo general realizado en este sector. En este sentido, Aidar (2015), expresa que las dos capacidades a analizar son los recursos fuerza laboral física y humana, y estructuras organizativas, formadas por el rol de las personas que operan en el quirófano, la estructura formal, los sistemas de planificación formales e informales, el control y la coordinación.

\section{Gestión hospitalaria y centro quirúrgico}

La nueva realidad organizacional muestra que el camino hacia el éxito se vuelve relevante a través del liderazgo diferenciado, el uso de la creatividad y una mayor competitividad. Para que esto ocurra, es necesario crear nuevos procesos y técnicas de gestión observando los valores internos, la misión y los objetivos organizacionales del hospital.

La Tabla 1 muestra investigaciones relevantes sobre el tema:

El quirófano, según Vogt (2010), es una unidad muy compleja del entorno hospitalario porque, además de las cirugías, se realizan pruebas de diagnóstico, análisis clínicos, patologías, administración de medicamentos y postcirugía, es decir, donde el paciente se recupera de la anestesia. Por lo tanto, el rendimiento de un quirófano está relacionado con la calidad de sus propios procesos y todos los servicios que lo respaldan, o sea, una combinación de equipo apropiado, instalaciones 
Tabla 1. Referencia teórica sobre gestión relacionada con centros quirúrgicos.

\begin{tabular}{|c|c|}
\hline Fuente & Objeto de Investigación \\
\hline Aidar (2015). M. M. Planificación estratégica y competitividad en salud. & $\begin{array}{l}\text { Proponer soluciones administrativas a los problemas que enfrenta el Siste- } \\
\text { ma de Salud de Brasil. }\end{array}$ \\
\hline $\begin{array}{l}\text { Amaral (2013) El proceso de planificación estratégica del Hospital Universitario de la Uni- } \\
\text { versidad Federal de Santa Catarina: entre intención y práctica. }\end{array}$ & $\begin{array}{l}\text { Planificación estratégica de un hospital universitario en el estado de Santa } \\
\text { Catarina y el resultado después de la implementación. }\end{array}$ \\
\hline $\begin{array}{l}\text { Assis (2017). La planificación estratégica de un Hospital Universitario Federal y su papel } \\
\text { regional en las políticas de salud pública. }\end{array}$ & $\begin{array}{l}\text { Propuesta de planificación estratégica de un hospital universitario en la ciu- } \\
\text { dad de Curitiba realizada en la Universidad Tecnológica Federal de Paraná. }\end{array}$ \\
\hline $\begin{array}{l}\text { Gomes (2009). A. Organización y gestión del Centro Quirúrgico de un Hospital Universi- } \\
\text { tario de Belo Horizonte - Minas Gerais, } 2009 .\end{array}$ & $\begin{array}{l}\text { La construcción de un conjunto de indicadores como herramienta de mo- } \\
\text { nitoreo en organizaciones de salud: una aplicación en un centro quirúrgico. }\end{array}$ \\
\hline $\begin{array}{l}\text { Brasil. Ministerio de Salud, Departamento de Vigilancia Sanitaria, Departamento de En- } \\
\text { fermedades No Transmisibles y Vigilancia de Enfermedades y Promoción de la Salud } \\
\text { (2017). Vigilancia de factores de riesgo y protección para enfermedades crónicas por en- } \\
\text { cuesta telefónica: estimaciones de frecuencia y distribución sociodemográfica de facto- } \\
\text { res de riesgo y protección para enfermedades crónicas en las capitales de los } 26 \text { estados } \\
\text { brasileños y el distrito federal en } 2017 \text {. }\end{array}$ & $\begin{array}{l}\text { Para verificar qué enfermedades crónicas del envejecimiento promueven la } \\
\text { necesidad de cirugía. }\end{array}$ \\
\hline $\begin{array}{l}\text { Vogt y Freitas (2012). Gestión organizacional en un centro quirúrgico de un hospital en } \\
\text { el Gran Porto Alegre. }\end{array}$ & $\begin{array}{l}\text { Investigar y analizar la gestión organizativa y el funcionamiento de un cen- } \\
\text { tro quirúrgico. }\end{array}$ \\
\hline $\begin{array}{l}\text { Vogt (2012). Gestión organizacional en un centro quirúrgico de un hospital en el Gran } \\
\text { Porto Alegre. }\end{array}$ & $\begin{array}{l}\text { Investigar y analizar la gestión y operación organizacional de un centro qui- } \\
\text { rúrgico. }\end{array}$ \\
\hline
\end{tabular}

Fuente: elaboración propia. Los títulos fueron traducidos por los autores.

apropiadas, tecnología, mano de obra calificada, etc.

\section{Indicadores de gestión}

Para mejorar el desempeño del quirófano ejecutando nuevos procesos, una forma viable es aplicar indicadores para monitorear la productividad, revisar procesos que fallaron y así desarrollar mejoras. Los indicadores son herramientas de monitoreo (Kaplan y Norton, 1992; 2000), para ayudar al proceso de gestión de las organizaciones de salud para la toma de decisiones, mejorando así la calidad de los servicios prestados.

Los indicadores cuantitativos se utilizan para obtener información numérica, determinar opiniones a través de encuestas y rastrear un historial de información, mientras que los cualitativos para demostrar las cualidades de los procesos de trabajo. En los dos tipos es posible justificar la necesidad de adquirir nuevas tecnologías, materiales, equipos, además de la adecuación y capacitación de los equipos involucrados en el trabajo del quirófano y sus alrededores, haciendo posible el control, la revisión y la evaluación de procesos y resultados.

La definición de indicadores, por lo tanto, debe ser un proceso dinámico que permita que todos los involucrados participen en la búsqueda de un equilibrio de intereses, convirtiéndolos en un instrumento de evaluación general del área. Este proceso supone que, si un indicador es importante, el consenso de los entrevistados será general y también generará el compromiso de todos los in- volucrados. Una tarea importante del proyecto de diseño de indicadores es evitar perder tiempo, recursos humanos/tecnológicos en controles y mediciones no necesarios en la sala de operaciones.

Con respecto al sector de Contraloría, Costenaro y Brondani (2012) creen que debe contar con la mayor cantidad de información a fin de obtener un mayor control económico y financiero de la organización, siendo necesario que todos los sistemas de información gerencial estén interconectados, permitiendo una gestión productiva y competente. Asimismo, Rezende (2003), afirma que la información se puede obtener a través de procesos de medición continuos e integrados, con el objetivo de elaborar y ejecutar procesos de información. Entonces, a través del monitoreo es posible generar información para promover cambios organizacionales de manera más rápida y con menores costos.

El Centro Quirúrgico, según Okano y Castilho (2007), consiste en una unidad con características específicas y equipos diferenciados, que brinda un servicio individualizado vinculado directa o indirectamente a las cirugías, por lo tanto, las estrategias de reducción de costos no son suficientes, y también es necesario comprender todo el funcionamiento de la estructura para desarrollar estrategias que justifiquen la importancia de las actividades.

Para Nepote, Monteiro y Hardy (2009), este es el revés que encuentra la gestión, porque la fun- 
cionalidad del quirófano es una de las principales medidas de eficiencia de una organización hospitalaria, requiriendo un aumento de su capacidad para atender a la población sin exceder el servicio.

Por su parte y complementando los párrafos anteriores, las autoras Nogueira y Castilho (2016), realizaron una investigación cuantitativa muy relevante con el objetivo de mapear y validar los subprocesos de la gestión de Residuos de Servicios de Salud en un Centro Quirúrgico, además de calcular el costo de materiales. La investigación destaca la relevancia de identificar el costo total promedio por cirugía.

La Tabla 2 muestra investigaciones sobre indicadores relacionados con los Centros Quirúrgicos:

Tabla 2. Referencia teórica sobre gestión relacionada con centros quirúrgicos.

\begin{tabular}{|c|c|}
\hline Fuente & Objeto de Investigación \\
\hline Giacomeli (2018). Uno de cada tres jóvenes empezó a beber entre los 11 y los 15 años. & Identificar las enfermedades crónicas que desencadenan la cirugía. \\
\hline $\begin{array}{l}\text { Marquez (2016). Construyendo un conjunto de indicadores como herramienta de moni- } \\
\text { toreo en organizaciones de salud: una aplicación en una sala de operaciones. }\end{array}$ & $\begin{array}{l}\text { Construyendo un conjunto de indicadores como herramienta de monitoreo en } \\
\text { organizaciones de salud: una aplicación en una sala de operaciones. }\end{array}$ \\
\hline $\begin{array}{l}\text { Nepote (2003). Análisis del desempeño de las actividades de la sala de operaciones a } \\
\text { través de indicadores cuantitativos y cualitativos. }\end{array}$ & $\begin{array}{l}\text { La construcción de un conjunto de indicadores como herramienta de moni- } \\
\text { toreo en organizaciones de salud: una aplicación en una sala de operaciones. }\end{array}$ \\
\hline $\begin{array}{l}\text { Paula (2006). La mayoría de los brasileños son sedentarios desde la adolescencia. So- } \\
\text { ciedad Brasileña de Nefrología. }\end{array}$ & $\begin{array}{l}\text { Verificar qué patologías proporciona el estilo de vida sedentario y, en conse- } \\
\text { cuencia, promueve el aumento de las cirugías. }\end{array}$ \\
\hline Costenaro y Brondani (2012). Contraloría en instituciones hospitalarias. & Funcionamiento de las organizaciones hospitalarias según la Contraloría. \\
\hline Silva y Ribeiro (2016). Cestión de quirófano: identificación de residuos. & $\begin{array}{l}\text { Identificar los artículos médicos hospitalarios desperdiciados en el Centro } \\
\text { quirúrgico y las causas de estos desechos en un hospital público y docente. }\end{array}$ \\
\hline $\begin{array}{l}\text { Sociedade Beneficente Israelita Brasileira Albert Einstein. (2016b). Planificación estra- } \\
\text { tégica. }\end{array}$ & Estudio del funcionamiento interno del hospital y rotación de pacientes. \\
\hline $\begin{array}{l}\text { Sociedade Beneficente Israelita Brasileira Albert Einstein. (2016a). Elementos funda- } \\
\text { mentales del flujo del paciente en el hospital. }\end{array}$ & Estudio del funcionamiento interno del hospital y rotación de pacientes. \\
\hline $\begin{array}{l}\text { Weiser, Haynes, Molina, et al. (2015). Estimación del volumen global de cirugía en 2012: } \\
\text { una evaluación que respalda los mejores resultados de salud. }\end{array}$ & $\begin{array}{l}\text { Estudio de las tasas quirúrgicas y los resultados de salud de la población } \\
\text { mundial. }\end{array}$ \\
\hline $\begin{array}{l}\text { Rose, Weiser, Hider, Wilson, Gruen \& Bickler (2015). Necesidad estimada de cirugía en } \\
\text { todo el mundo basada en la prevalencia de enfermedades: una estrategia de modelado } \\
\text { para la Estimación Global de Salud de la OMS. } 2015 .\end{array}$ & $\begin{array}{l}\text { Volumen global mínimo estimado de necesidad quirúrgica para tratar enfer- } \\
\text { medades epidemiológicas prevalentes en } 21 \text { regiones del Clobal Burden of } \\
\text { Disease Study (CBD). }\end{array}$ \\
\hline
\end{tabular}

Fuente: elaboración propia. Los títulos fueron traducidos por los autores.

En tanto, Marquez (2016), concluyó su disertación de Maestría Profesional en Cestión de Organizaciones de Salud de la Facultad de Medicina, explorando formas de construir un conjunto de indicadores para el seguimiento de un centro quirúrgico, a través de una investigación de campo exploratoria, planteando datos primarios y secundarios, utilizando una herramienta de análisis de procesos jerárquicos (AHP), propuesta por Saaty (1991, como se citó en Marquez, 2016), cuyo enfoque fue la definición de las ponderaciones de importancia relativa de los indicadores. Su estudio no se centró en el centro quirúrgico; pero trajo aportes relevantes que son útiles para la construcción de esta investigación.
Nepote (2003), a su vez, desarrolló un estudio sobre el análisis del desempeño de actividades en el quirófano, siendo uno de los pocos trabajos de gestión con delimitación en esta temática. A través de indicadores cuantitativos y cualitativos, se muestra un análisis sistemático de los datos utilizados por el personal de enfermería en un hospital privado de tamaño medio, y posibilita mediante el análisis mensual de los indicadores identificar la necesidad de nuevas tecnologías, actualizaciones de equipos y la adecuación y capacitación del personal, buscando la gestión organizacional, control y revisión de todos los procesos internos, como también, analizando los resultados. 
En similar línea enunciativa, Comes (2009), presentó su disertación con el tema Organización y Gestión del Centro Quirúrgico de un Hospital Universitario de Belo Horizonte, desarrollando un programa que garantizó calidad, eficiencia y resultados en el Centro Quirúrgico del Hospital das Clínicas de la Universidad Federal de Minas Gerais, que comprende un proceso de gestión. Destacó los factores que facilitaron y dificultaron el proceso de trabajo y las relaciones entre los diferentes profesionales que trabajaron en el Centro Quirúrgico; realizó un estudio de caso cualitativo, recolectando los datos a través de entrevistas, teniendo los resultados analizados y sometidos a análisis para la construcción de un nuevo modelo de gestión.

\section{METODOLOCÍA}

Al recopilar datos a través de la investigación bibliográfica sobre el tema, se creó un cuestionario con posibles indicadores para la validación o propuesta de nuevos indicadores por parte de los empleados de la institución estudiada, con el fin de investigar los factores relevantes que aumentan la rotación y la productividad del Centro Quirúrgico.
Este es un estudio de caso cualitativo, para el cual se elaboró un cuestionario con el fin de proponer indicadores para la gestión y seguimiento de las actividades, buscando controlar los costos y mejorar los resultados.

Blanck y Bandeira (2015), investigaron la capacidad operativa de un centro quirúrgico y señalan que, a diferencia de otros estudios, el diferencial de esta investigación es modelar la asignación en función de las características presentes en los ambientes del Centro Quirúrgico. Este hecho destaca que el Centro Quirúrgico está muy poco investigado y esto dificulta que un mayor número de autores construya la metodología. Luego se observa que son pocos los investigadores que abordan el tema de gestión que enfrenta el Centro Quirúrgico, por consiguiente, para la elaboración del instrumento se consideraron lo aportes teóricos y empíricos de Nepote y cols. (2009).

Posteriormente, se desarrolló un instrumento en forma de cuestionario para validar los indicadores; los encuestados firmaron un Término de Consentimiento Libre e Informado para garantizar el proceso ético de solicitud. Los indicadores planteados a través de la literatura se propusieron para componer en el futuro, un panel de monitoreo de la unidad del Centro Quirúrgico, como se muestra en la Figura 1:
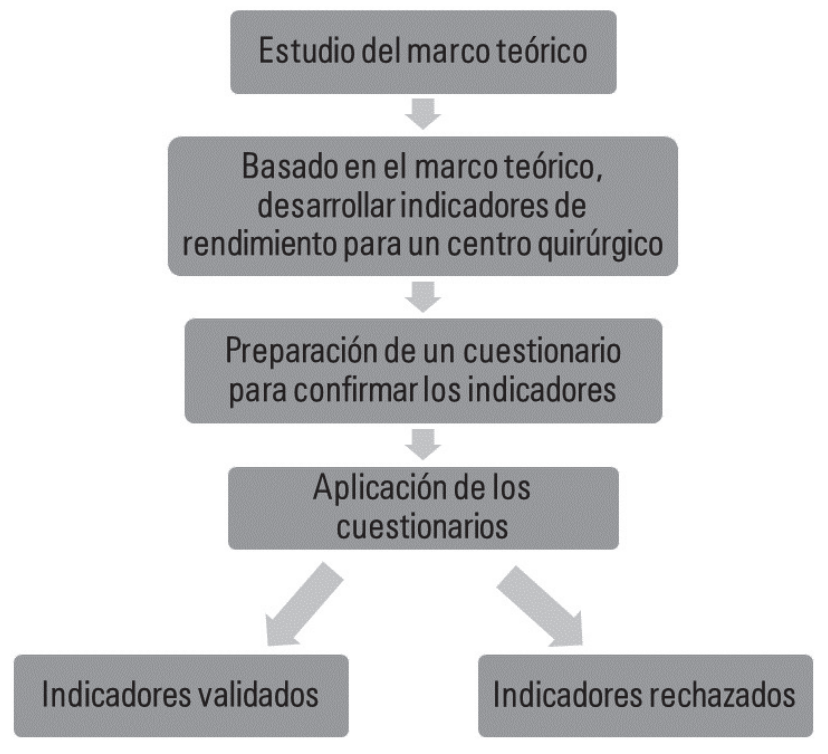

Figura 1. Organización del trabajo. Fuente: elaboración propia. 
Siguiendo el objetivo propuesto, la secuencia metodológica fue:

- Identificar qué indicadores, a través de la literatura, deberían ser determinados y su importancia para el mejor funcionamiento del Centro Quirúrgico; expansión del número de cirugías y mejores resultados en el desempeño de los equipos;

- Preparar un cuestionario para confirmar los indicadores mediante una encuesta;

- Seleccionar los indicadores validados que deben ser monitoreados a través del resultado del cuestionario preparado.

Los procedimientos metodológicos utilizados para apoyar este estudio se categorizan como exploratorios, a través de la investigación bibliográfica y la investigación participativa. El enfoque cualitativo se utilizó para analizar los datos que se recopilaron mediante un cuestionario distribuido al personal del Centro Quirúrgico del HCFMB, basado en indicadores a través de una reunión el 19 de febrero de 2019, para confirmación de qué indicadores se ade- cuan al Centro Quirúrgico, donde estuvieron presentes un representante de control de calidad, uno del sector de TI, el jefe de la sala de operaciones, dos enfermeras, el jefe de enfermería y el director clínico del hospital, por lo tanto, siete personas. Los empleados fueron seleccionados por su conocimiento, su capacitación y experiencia en la sala de operaciones.

Entonces, por tratarse de un conocimiento técnico específico, se eligió una población cualitativa de participantes. Luego de la presentación del proyecto y el cuestionario, se eliminaron algunos indicadores que no se ajustaban a la realidad del hospital o porque ya cuentan con algún tipo de seguimiento: como tasas de ocupación, tasas de cancelación de cirugías, tasas de cirugías sin programación previa, tasa de ocupación por tamaño quirúrgico.

Según el equipo que formó la reunión, los indicadores relacionados con el tiempo son más relevantes, mientras que los indicadores de desempeño no son importantes para observar.

La Tabla 3 resume los indicadores propuestos:

Tabla 3. Indicadores propuestos.

\begin{tabular}{|c|c|c|}
\hline & Indicadores & Fuente \\
\hline 1 & Retraso en equipos quirúrgicos y anestesistas & Gomes (2009) \\
\hline 2 & Número promedio de cirugías por día & Nepote (2003) \\
\hline 3 & Duración promedio de la cirugía más 20 minutos de limpieza & Nepote (2003) \\
\hline 4 & Tasa de ocupación del Centro Quirúrgico & Nepote (2003) \\
\hline 5 & Tasa de ocupación por período & Nepote (2003) \\
\hline 6 & Tasa de ocupación por tamaño quirúrgico & Nepote (2003) \\
\hline 7 & Tasa de cirugías que duraron el tiempo programado & Nepote (2003) \\
\hline 8 & Tasa de cancelación de cirugía & Nepote (2003) \\
\hline 9 & Tasa de cirugías sin programación previa (ajuste, urgencia, emergencia) & Nepote (2003) \\
\hline 10 & Tasa de cirugías iniciadas a la hora programada & Nepote (2003) \\
\hline 11 & Tasa de espera satisfactoria entre una cirugía y otra & Nepote (2003) \\
\hline 12 & Dinámica general del quirófano & Nepote (2003) \\
\hline 13 & Llegada tardía del paciente al hospital & Nepote (2003) \\
\hline 14 & Recepción del Hospital & Nepote (2003) \\
\hline 15 & Servicio de hospitalización (salas y ambulatorio) & Nepote (2003) \\
\hline 16 & Recolección de pruebas subsidiarias, electrocardiograma, etc. & Nepote (2003) \\
\hline 17 & Esperando el efecto de la medicación & Nepote (2003) \\
\hline 18 & CC retraso debido a la falta de capacitación o personal & Nepote (2003) \\
\hline 19 & Momento del proceso de anestesia y comienzo de la cirugía & Nepote (2003) \\
\hline 20 & Preparación del paciente en OS & Nepote (2003) \\
\hline 21 & Retraso en el transporte de pacientes a CC & Nepote (2003) \\
\hline 22 & Falta de materiales y equipos & Nepote (2003) \\
\hline 23 & Falta de productos sanguíneos en el banco de sangre & Nepote (2003) \\
\hline 24 & Tasa de infección del sitio quirúrgico & Marquez (2016) \\
\hline 25 & Tiempo promedio de limpieza del quirófano & Marquez (2016) \\
\hline 26 & Tiempo promedio del paciente entre el final del proceso quirúrgico y la salida del paciente del quirófano & Marquez (2016) \\
\hline
\end{tabular}




\section{RESULTADOS Y DISCUSIONES}

Para la validación de los indicadores, como se describe en la sección anterior, utilizamos profesionales del hospital que fueron seleccionados por su conocimiento y experiencia con el objeto de la investigación, en este caso, el quirófano.
La Tabla 4 presenta la síntesis de lo que se discutió y cambió a partir de la propuesta inicial, clasificándose como rechazado o validado de acuerdo con las respuestas. Se observa que el rechazo no significa que el indicador no sea recomendado para otros Centros Quirúrgicos, sino que ya estaba considerado en la realidad del HCFMB.

Tabla 4. Validación de Indicadores.

\begin{tabular}{|c|c|c|c|}
\hline & Indicadores & Fuente & Justificación \\
\hline 1 & Retraso de los equipos quirúrgicos y del anestesista & Gomes 2009) & Debe medirse para obtener la optimización del tiempo. \\
\hline 2 & Promedio de Cirugías por día. & Nepote (2003) & Debe medirse para obtener la optimización del tiempo. \\
\hline 3 & Duración media de la cirugía más 20 min de limpieza. & Nepote (2003) & $\begin{array}{l}\text { Corregido por } 30 \text { min. tiempo de limpieza, y pretenden disminuir y op- } \\
\text { timizar este tiempo. }\end{array}$ \\
\hline 4 & Tasa de ocupación del Centro Quirúrgico. & Nepote (2003) & Medida requerida para la optimización del tiempo. \\
\hline 5 & Tasa de ocupación por períodos. & Nepote (2003) & No existe tal división por períodos. \\
\hline 6 & Tasa de ocupación para tamaños quirúrgicos. & Nepote (2003) & $\begin{array}{l}\text { Debe medirse para obtener la optimización del tiempo. } \\
\text { Nota: Pronto no habrá más cirugías menores, porque serán transferi- } \\
\text { das a otra sala de operaciones. }\end{array}$ \\
\hline 7 & Tasa de cirugías que duraron el tiempo programado. & Nepote (2003) & $\begin{array}{l}\text { En HC no hay cirugía programada, solo la primera cirugía. Quieren im- } \\
\text { plementar la programación. }\end{array}$ \\
\hline 8 & Tasa de cancelación de cirugía. & Nepote (2003) & Medida ya realizada en el hospital. \\
\hline 9 & $\begin{array}{l}\text { Tasa de cirugías sin programación previa (ajuste, urgen- } \\
\text { cia, emergencia). }\end{array}$ & Nepote (2003) & $\begin{array}{l}\text { En HC no hay cirugía programada, solo la primera cirugía. Su objetivo es } \\
\text { implementar la programación. }\end{array}$ \\
\hline 10 & La tasa de cirugías que empezaron a la hora programada. & Nepote (2003) & $\begin{array}{l}\text { En HC no hay cirugía programada, solo la primera cirugía. Quieren im- } \\
\text { plementar la programación. }\end{array}$ \\
\hline 11 & Tasa de espera satisfactoria entre una cirugía y otra. & Nepote (2003) & $\begin{array}{l}\text { Análisis válido con el objetivo de reducir el tiempo de espera entre una } \\
\text { cirugía y otra, en el que el promedio actual es de } 30 \text { min. }\end{array}$ \\
\hline 12 & Dinámica general del quirófano. & Nepote (2003) & No se identificó como medida de comunicación de profesionales. \\
\hline 13 & Retraso en la llegada del paciente al hospital. & Nepote (2003) & $\begin{array}{l}\text { Relevante solo para cirugías medianas y pequeñas, porque en las gran- } \\
\text { des, el paciente es hospitalizado el día antes de la cirugía. }\end{array}$ \\
\hline 14 & Recepción en el hospital. & Nepote (2003) & No hay forma de medir la comunicación y el rendimiento. \\
\hline 15 & $\begin{array}{l}\text { Servicio para pacientes hospitalizados (salas y pacientes } \\
\text { ambulatorios). }\end{array}$ & Nepote (2003) & No hay forma de medir la comunicación y el rendimiento. \\
\hline 16 & $\begin{array}{l}\text { Recolección de pruebas subsidiarias, electrocardiograma, } \\
\text { etc. }\end{array}$ & Nepote (2003) & $\begin{array}{l}\text { La ubicación del laboratorio favorece la recogida de exámenes y no hay } \\
\text { necesidad de calcular este indicador. }\end{array}$ \\
\hline 17 & La espera del efecto de la medicación. & Nepote (2003) & El equipo no consideró esta medida relevante. \\
\hline 18 & $\begin{array}{l}\text { Retraso en el CC debido a la falta de capacitación o per- } \\
\text { sonal. }\end{array}$ & Nepote (2003) & No hay forma de medir el rendimiento. \\
\hline 19 & $\begin{array}{l}\text { Momento del proceso de anestesia y comienzo de la ci- } \\
\text { rugía. }\end{array}$ & Nepote (2003) & El equipo no consideró esta medida relevante. \\
\hline 20 & Preparación del paciente en OS. & Nepote (2003) & Debe medirse para obtener la optimización del tiempo. \\
\hline 21 & Retraso en el transporte del paciente al CC. & Nepote (2003) & Debe medirse para obtener la optimización del tiempo. \\
\hline 22 & Falta de materiales y equipos. & Nepote (2003) & Debe medirse para obtener la optimización del tiempo. \\
\hline 23 & Falta de productos sanguíneos en el banco de sangre. & Nepote (2003) & No es necesario ya que no es un problema de la realidad del CC. \\
\hline 24 & Tasa de infección nosocomial en sitio quirúrgico. & Marquez (2016) & Medida ya realizada en el hospital. \\
\hline 25 & Tiempo medio de limpieza del quirófano. & Marquez (2016) & $\begin{array}{l}\text { Son } 30 \text { minutos. Tiempo que desean optimizar a través de la factu- } \\
\text { ración. }\end{array}$ \\
\hline 26 & $\begin{array}{l}\text { Tiempo promedio del paciente entre el final del proceso } \\
\text { quirúrgico y la salida del paciente del quirófano. }\end{array}$ & Marquez (2016) & Necesita medir para obtener la optimización del tiempo. \\
\hline
\end{tabular}

Fuente: elaboración propia. 
A continuación, se explica cada indicador validado, según los profesionales de HC Botucatu:

1. Retraso de los equipos quirúrgicos y del anestesista: retraso medio de las cirugías debido al retraso de los profesionales, y estar al tanto de los posibles motivos de retraso para una corrección adecuada;

2. Indicador promedio de cirugías por día: evalúa si el promedio de cirugías por día es satisfactorio para mantener la calidad de los servicios prestados con el número de habitaciones disponibles en el Centro Quirúrgico y para cumplir con los objetivos de ocupación del sitio. Después de la planificación, es posible analizar el crecimiento en el número de cirugías realizadas;

3. La duración promedio de la cirugía más 20 minutos. Limpieza: este tiempo se establece sobre la base de estudios históricos del intervalo de tiempo entre una cirugía y otra, en la misma habitación y el mismo cirujano. Cuando el tiempo esperado es mayor, será necesario reubicarlo desde la sala de operaciones. Rotación de habitaciones para un mayor uso del tiempo y el uso del espacio disponible;

4. Tasa de ocupación del Centro de Cirugía: Calcula el tiempo total inactivo de salas de operaciones por día, teniendo en cuenta que debe haber tiempo disponible para la atención urgente y de emergencia, y las cirugías de adaptación. Para medir la ociosidad, se debe considerar el tiempo requerido para la limpieza y la organización del Centro Quirúrgico, y el tiempo de preparación para los instrumentos;

5. La tasa de ocupación por tamaño quirúrgico es un indicador que puede mostrar el tiempo dedicado según el tamaño de cada cirugía, que puede ser pequeña, mediana o grande;

6. La tasa de cirugías que duraron el tiempo programado se clasificará y se dividirá en: porcentaje de cirugía completada y porcentaje de cirugía perdida. Puede mostrar la necesidad de simplificar el mapa quirúrgico y evitar la inactividad de las salas de operaciones; también puede ser importante conocer las características de los equipos quirúrgicos cuando se trata del tiempo de uso del espacio.

7. La tasa de cancelación de cirugía se puede dividir en:

- Cirugías totales;

- Razones hospitalarias;
- Razones fuera del hospital;

- Razones relacionadas con el paciente;

A través de este indicador, se puede verificar si existe la necesidad de confirmar datos como alojamiento, cama, desglose de equipos, mantenimiento de equipos de rutina, cancelación de cirugías por parte del paciente por no tener condiciones clínicas o no asistir.

8. La tasa de cirugías no programadas (ajuste, urgencia, emergencia), puede mostrar si realmente hay urgencia y emergencia para la mejor organización de la industria;

9. La tasa de cirugías iniciadas a la hora programada puede indicar retrasos del personal, retrasos de los exámenes o incluso retrasos de los pacientes. A través de esta verificación es posible detectar las razones de los retrasos y hacer posibles correcciones;

10. La tasa de espera entre una cirugía y otra indica si el servicio de enfermería y limpieza de las habitaciones puede tener una mayor velocidad;

11. Cuando el paciente produce el retraso, también puede ser necesario reubicar las cirugías que seguirán después de un retraso de 20 minutos. La demora preoperatoria es un indicador que evalúa la necesidad de una visita preanestésica por adelantado y solicita al paciente que traiga todos los exámenes preoperatorios y la evaluación clínica previamente publicada para la cirugía;

12. La preparación del paciente en el sistema operativo se refiere principalmente a la cirugía plástica y la cirugía de venas varicosas, en las cuales el cirujano delimita el área con su propia pluma en el sistema operativo. A través de este indicador es posible medir el tiempo empleado en la preparación del paciente, y si este proceso se realiza fuera del SO contribuye a un menor tiempo de utilización del espacio;

13. Retraso del transporte del paciente al centro quirúrgico: tiempo medio de retraso del transporte del paciente al sistema operativo, debido a la falta de comunicación entre los equipos y al número insuficiente de profesionales que realizan este proceso;

14. Falta de materiales y equipo: mide el número de cirugías interrumpidas debido a una falla imprevista del equipo o la falta de equipo esterilizado sobre la mesa; 
15. Tasa de infección del sitio quirúrgico: mide la frecuencia de complicaciones debido a una infección hospitalaria, lo que hace que el paciente regrese al quirófano;

16. Tiempo promedio de limpieza de la sala de operaciones: mide el tiempo empleado en el proceso de limpieza y esterilización del sistema operativo, verificando si es necesario reubicar la sala y si la cantidad de personal que realiza este trabajo es suficiente;

17. Tiempo promedio entre el final del proceso quirúrgico y la salida del paciente del quirófano: verificar si la disponibilidad de espacio es suficiente para aumentar el número de cirugías;

Estos indicadores se refieren específicamente a la realidad del Centro Quirúrgico del Hospital Botucatu. Por consiguiente, el resultado obtenido se aplica solo a él. El uso de los indicadores propuestos en este estudio, debe ser revalidado de acuerdo con la realidad de la sala de operaciones del hospital para ser optimizado.

\section{CONSIDERACIONES FINALES}

A través de la literatura se construyó una estructura de indicadores en la que algunos de ellos fueron validados de acuerdo con la realidad del HCFMB, lo que demuestra que hubo divergencias entre la práctica y la literatura, porque cada institución tiene su especificidad.

La estructura de indicadores construida tenía un total de 26 indicadores como propuesta inicial y, al final de la investigación, 17 indicadores fueron validados y nueve fueron rechazados.

Según la investigación, lo que dificulta el servicio en el Centro Quirúrgico para que se produzca un mayor rendimiento en el número de cirugías y, en consecuencia, disminuya la lista de espera, es el tiempo empleado en cada procedimiento y su medición por indicadores validados.

Según los resultados obtenidos en la reunión a través del cuestionario, los indicadores relacionados con el tiempo son de mayor importancia y se adaptan mejor a la realidad del hospital.

Las propuestas futuras se refieren a la construcción de una tabla de rotación, que requiere el conocimiento de datos importantes para su construcción como las horas disponibles de cada profesional, la especialidad y las posibles cirugías 102 realizadas por cada profesional, el tiempo promedio de cada cirugía desarrollada y la creación de software para el desarrollo de este gráfico de rotación y actualización diaria, de acuerdo con los datos y las condiciones ingresadas.

En definitiva, con la rotación se puede eliminar el tiempo de espera entre una cirugía y otra, que es el tiempo que el mismo médico espera para empezar la próxima intervención.

\section{REFERENCIAS}

Aidar, M. M. (2015). Planejamento estratégico e competitividade na saúde. São Paulo: Saraiva, 2015.

Amaral, H. C. M. (2013). O processo de planejamento estratégico do hospital universitário da Universidade Federal de Santa Catarina: entre a intenção e a prática. Dissertação (Mestrado) - Universidade Federal de Santa Catarina, SC, Brasil.

Assis, L. (2017). O planejamento estratégico de um hospital universitário federal e sua atuação regional nas políticas públicas de saúde. Dissertação (Mestrado) - Universidade Tecnológica Federal do Paraná, Curitiba, PR, Brasil.

Blanck, M., y Bandeira, D. L. (2016). Analysis of the operational capacity of a surgical center: mathematical modelling applied to scaling and resource allocation. REGE - Revista De Cestão, 22(4), 565-583.

Brasil. Ministério da Saúde. Secretaria de Vigilância em Saúde. (2017). Departamento de Vigilância de Doenças e Agravos não Transmissiveis e Promoção da Saúde. Vigilância de fatores de risco e proteção para doenças crônicas por inquérito telefônico: estimativas sobre frequência e distribuição sociodemográfica de fatores de risco e proteção para doenças crônicas nas capitais dos 26 estados brasileiros e no distrito federal em 2017. Brasília: Ministério da Saúde. Recuperado de: http:// bvsms.saude.gov.br/bvs/publicacoes/vigitel_ brasil_2017_vigilancia_fatores_riscos.pdf

CISA - Centro de Informações sobre Saúde e Álcool. (2018). Estatísticas Mundiais de Saúde. São Paulo: Cisa. Recuperado de: http://www. cisa.org.br/artigo/9682/estatisticas-mundiais-saude-2018.php 
Costenaro, A., y Brondani, G. (2012). A controladoria em instituições hospitalares. Revista Eletrônica de Contabilidade, 2(2), 1-18.

Damasio, M. (2016). Projeto de lei. São Paulo: Alesp, 30 de Junio de 2016. Sao Paulo: Alesp. Recuperado de: https://www.al.sp.gov.br/ propositura/?id=1323005

Giacomeli, C. 1 (2018). Um cada 3 jovens começou a beber entre os 11 e os 15 anos. Metro Jornal, 2 abr. São Paulo, Brasil. Recuperado de: https://www.metrojornal.com.br/foco/2018/04/02/1-em-cada-3-jovens-comecou-a-beber-entre-os-11-e-os-15-anos.html

Gomes, M. C. S. M. A. (2009). Organização e Gestão do Centro Cirúrgico de um Hospital Universitário de Belo Horizonte - Minas Gerais. Dissertação (Mestrado) - Universidade Federal de Minas Gerais, Belo Horizonte.

HCFMB - Hospital das Clínicas da Faculdade de Medicina de Botucatu. (2017). Vídeo Institucional. Recuperado de: http://www.hcfmb. unesp.br/ el 12 de Julio de 2018.

Kaplan, R. S., y Norton, D. P. (1992). The balanced scorecard - measures that drive performance. Harvard Business Review, 70, 71-79.

Marquez, P. B. (2016). A construção de um conjunto de indicadores como ferramenta de monitoramento em organizações de saúde: uma aplicação em um centro cirúrgico Dissertação (Mestrado) - Universidade de São Paulo, Ribeirão Preto, SP, Brasil.

Mastrantonio, M. A. y Graziano, K.U. (2002). Proposta de um instrumento de avaliação dos padrões de qualidade de uma unidade de centro cirúrgico ajuizado por especialistas. 0 mundo da Saúde. São Paulo, 26(2), 332-41.

Nepote, M. H. A. (2003). Análise do desempenho das atividades no centro cirúrgico através de indicadores quantitativos e qualitativos. Revista de Administração em Saúde, 5, 21-30.

Nepote, M. H. A., Monteiro, I. U., y Hardy, E. (2009). Association between operational indexes and the utilization rate of a general surgery center. Revista Latino-Americana de Enfermagem, 17(4), 529-534.

Nogueira, D. N. G., y Castilho, V. (2017). Resíduos de serviços de saúde: mapeamento de processo e gestão de custos como estratégias para sustentabilidade em um centro cirúrgico. REGE - Revista De Gestão, 23(4), 362-374.
Okano, H.I.H. y Castilho,V. (2007). Levantamento do custo do processo admissional de um técnico de enfermagem de um hospital de ensaio. Revista da Escola de Enfermagem da USP, 41(3), 492-499.

OMS - Organização Mundial da Saúde. (2017). Cigarro mata mais de 5 milhões de pessoas, segundo OMS. Recuperado de: www.brasil. gov.br/noticias/saude/2014/08/cigarro-mata-mais-de-5-milhoes-de-pessoas-segundooms

Paradella, R. (2018). Número de idosos cresce 18\% em 5 anos e ultrapassa 30 milhões em 2017. Agência de Notícias IBGE. Recuperado de: https://agenciadenoticias.ibge.gov.br/ agencia-noticias/2012-agencia-de-noticias/ noticias/20980-numero-de-idosos-cresce-18-em-5-anos-e-ultrapassa-30-milhoesem-2017

Paula, R. B. (2006). IBGE: Maioria dos brasileiros é sedentária a partir da adolescência. Sociedade Brasileira de Nefrologia. Sociedade Brasileira de Nefrologia, 2006. Recuperado de: https:// sbn.org.br/publico/doencas-comuns/o-excesso-de-peso-e-suas-consequencias/

Rezende, J. F. C. (2003). Balanced Scorecard e a Gestão do Capital Intelectual: Alcançando a performance balanceada na economia do conhecimento. Rio de Janeiro: Elsevier.

Rose, J., Weiser, T. G, Hider, P, Wilson, L., Gruen, R.L., y Bickler, S. W. (2015). Estimated need for surgery worldwide based on prevalence of diseases: a modelling strategy for the WHO Global Health Estimate. Lancet Glob Health, 3(2), 513-520.

São Paulo. Assembleia Legislativa do Estado de São Paulo. (2016). Projeto de Lei $n^{0}$ 625/2016. São Paulo, Brasil. Recuperado de: www.al.sp. gov.br/propositura/?id=1000001152

Silva, M. J. N., y Ribeiro, A. L. (2016). Gestão em centro cirúrgico: identificação de desperdícios. Revista SOBECC, 21(2), 82-89.

Sociedade Beneficente Israelita Brasileira Albert Einstein. (2016a). Elementos Fundamentais o Fluxo do Paciente no Hospital. São Paulo, Einstein. Recuperado de: www.einstein.br/ empresas-hospitais/consultoria-gestao/artigos/elementos-fundamentais-fluxo-paciente-hospital 
Sociedade Beneficente Israelita Brasileira Albert Einstein. (2016b). Planejamento estratégico. São Paulo: Einstein. Recuperado de: https:// www.einstein.br/empresas-hospitais/consultoria-gestao/servicos/planejamento-estrategico

Vogt, R. M. (2010). Gestão Organizacional em um Centro Cirúrgico de um Hospital da Grande Porto Alegre. Trabalho de conclusão de curso (Especialização em Gestão Empresarial) - Universidade Feevale, Novo Hamburgo. Recuperado de: https://pt.scribd.com/doc/46829392/ Cestao-Organizacional-em-um-Centro-Cirurgico-de-um-Hospital-da-grande-Porto-Alegre-Romi-Maria-Vogt
Vogt, R. M. y Freitas, E. C. (2012). Cestão organizacional em um centro cirúrgico de um hospital da Grande Porto Alegre. Revista Cestão e desenvolvimento, 9(1), 33-46.

Weiser, T. G., Haynes, A. B., Molina, G., Lipsitz, S.R., Esquivel, M.M., Uribe-Leitz, T., Fu, R., Azad, T., Chao, T.E., Berry, W.R., Gawande, A.A. (2015). Estimate of the global volume of surgery in 2012: an assessment supporting improved health outcomes. The Lancet, 385, 511. 